\title{
Analysis of Genomic Regions Associated With Coronary Artery Disease Reveals Continent-Specific Single Nucleotide Polymorphisms in North African Populations
}

\author{
Daniela Zanetti ${ }^{1}$, Marc Via ${ }^{2}$, Robert Carreras-Torres ${ }^{1}$, Esther Esteban ${ }^{1}$, Hassen Chaabani ${ }^{3}$, \\ Fatima Anaibar ${ }^{4}$, Nourdin Harich ${ }^{4}$, Rachida Habbal ${ }^{5}$, Noreddine Ghalim ${ }^{6}$, and Pedro Moral ${ }^{1}$ \\ ${ }^{1}$ Department of Animal Biology-Anthropology, University of Barcelona, Barcelona, Spain \\ ${ }^{2}$ Department of Psychiatry and Clinical Psychobiology and Institute for Brain, Cognition and Behavior (IR3C), \\ Universitat de Barcelona, Barcelona, Spain \\ ${ }^{3}$ Laboratory of Human Genetics and Anthropology, Faculty of Pharmacy, University of Monastir, 5000 Monastir, Tunisia \\ ${ }^{4}$ Laboratoire des Sciences Anthropogénétiques, Faculté des Sciences, Université Chouaib Doukkali, El Jadida, Morocco \\ ${ }^{5}$ Centre de Cardiologie, CHU IBN ROCHD, Casablanca, Morocco \\ ${ }^{6}$ Laboratoire de Biochimie, Institut Pasteur du Maroc, Casablanca, Morocco
}

Received March 15, 2015; accepted August 21, 2015; released online January 16, 2016

Copyright (C) 2016 Daniela Zanetti et al. This is an open access article distributed under the terms of Creative Commons Attribution License, which permits unrestricted use, distribution, and reproduction in any medium, provided the original author and source are credited.

\begin{abstract}
Background: In recent years, several genomic regions have been robustly associated with coronary artery disease (CAD) in different genome-wide association studies (GWASs) conducted mainly in people of European descent. These kinds of data are lacking in African populations, even though heart diseases are a major cause of premature death and disability.

Methods: Here, 384 single nucleotide polymorphisms (SNPs) in the top four CAD risk regions $(1 \mathrm{p} 13,1 \mathrm{q} 41,9 \mathrm{p} 21$, and 10q11) were genotyped in 274 case-control samples from Morocco and Tunisia, with the aim of analyzing for the first time if the associations found in European populations were transferable to North Africans.

Results: The results indicate that, as in Europe, these four genetic regions are also important for CAD risk in North Africa. However, the individual SNPs associated with CAD in Africa are different from those identified in Europe in most cases (1p13, 1q41, and 9p21). Moreover, the seven risk variants identified in North Africans are efficient in discriminating between cases and controls in North African populations, but not in European populations.

Conclusions: This study indicates a disparity in markers associated to CAD susceptibility between North Africans and Europeans that may be related to population differences in the chromosomal architecture of these risk regions.
\end{abstract}

Key words: CAD genetic risk; North Africa; SNPs; haplotype blocks; genetic association

\section{INTRODUCTION}

Cardiovascular disease (CVD) continues to be the leading cause of mortality and morbidity in Western populations. ${ }^{1}$ According to World Health Organization data, 17.3 million people died from CVD in 2008, representing $30 \%$ of all global deaths (http://www.who.int/cardiovascular_diseases/ about_cvd/en/). In North Africa and the Middle East, noncommunicable diseases are an increasingly prevalent cause of premature death and disability (http://www.who.int/whr/2013/ report/en/). For example, ischemic heart disease and stroke have increased by $44 \%$ and $35 \%$, respectively, during the last 20 years.
The most common type of heart disease is coronary artery disease (CAD), which is a paradigm of complex disease, where environmental, lifestyle, and genetic factors interact to determine the clinical phenotype. ${ }^{2}$ In recent years, several genetic variants robustly associated with $\mathrm{CAD}$ have been detected, mainly in people of European descent (96\%), ${ }^{3}$ through genome-wide association studies (GWASs). Previous surveys in non-European populations have suggested that markers associated in one population may not always easily translate to other populations. Associations found in Europeans must be investigated in other ethnic groups $^{3}$ to assess the replicability of association signals or to detect new population-specific risk markers. For example, 
three variants in the major susceptibility gene (NOD2) for Crohn's disease were associated with susceptibility in Europeans. ${ }^{4,5}$ However, the same variants did not show evidence of association in Morocco and Tunisia. ${ }^{6,7}$ Likewise, the genetic susceptibility alleles for glaucoma in European populations do not seem to play a substantial role in populations of African ancestry. ${ }^{8}$ On the contrary, a recent study affirmed that Europeans and North Africans share the same 13 risk markers for type 2 diabetes. ${ }^{9}$ For the most studied CAD risk regions, 9p21, the lack of replication of European association signals in African samples was previously reported for coronary artery calcification ${ }^{10}$ and ischemic stroke. ${ }^{11}$ These differences in association signals between different population groups raise intriguing questions, and an increase in the number of epidemiological studies in different populations is required not only to validate the known risk loci, but also to identify new population-specific susceptibility variants.

In this way, the present study deals with a fine analysis of the four most validated CAD risk regions, $1 \mathrm{p} 13,1 \mathrm{q} 41,9 \mathrm{p} 21$, and $10 \mathrm{q} 11$, which are included among the labeled "top 12 golden loci", 2 in a novel set of case-control samples from North Africa. The main goal is to check the level (genomic regions and/or individual sites) to which the $\mathrm{CAD}$ associations previously found in Europe may also be transferable to North Africa, specifically to people of Tunisian and Moroccan origin. To accomplish this goal, 384 single nucleotide polymorphism (SNPs) on those four genomic regions were genotyped in case-control samples from North Africa. The associations and trends detected in North African samples were compared with available data from southern Europe. Finally, the combined effects (risk score) of the associated markers found in North Africa, and their ability to discriminate between cases and controls, were assessed.

\section{MATERIALS AND METHODS}

\section{Samples}

A total of 142 cases and 132 controls from Morocco and Tunisia (52\% males) were analyzed in the present study. The Moroccan samples consisted of 72 subjects from the area of Casablanca, along with 51 controls from the Doukkala-Abda region in west-central Morocco. The Tunisian case samples included 70 patients treated at the Department of Cardiology of the University Hospital Fattouma Bourguiba (Monastir, Tunisia). As a control group, 81 unrelated individuals, free from any $\mathrm{CAD}$ or related disorders, were randomly selected from the same large geographical area to which the patients belonged (the center of Tunisia). In both countries, CAD patients were diagnosed for ischemic heart disease complicated by myocardial infarction (MI), which was confirmed by electrocardiography and coronary angiography. All participants provided written informed consent, and the study was approved by the Ethics Committee of the University of Barcelona.
Table 1. Baseline characteristics of the samples

\begin{tabular}{llcccc}
\hline & & Morocco & Tunisia & Milan & Girona \\
\hline$N$ & Cases & 72 & 70 & 1693 & 312 \\
\multirow{2}{*}{ Mean Age (SD) } & Controls & 51 & 81 & 1668 & 317 \\
& Cases & $56.5(10.8)$ & $40.3(10.1)$ & $39.4(4.9)$ & $45.9(5.9)$ \\
\multirow{2}{*}{ Males } & Controls & $23.0(1.5)$ & $23.8(3.4)$ & $39.3(5.0)$ & $46.0(5.6)$ \\
& Cases & 68.0 & 55.7 & 88.3 & 79.5 \\
& Controls & 54.9 & 29.6 & 88.3 & 78.5 \\
\hline
\end{tabular}

$\mathrm{SD}$, standard deviation.

For comparative purposes, we used data from two southern European matched case-control samples (Milan, ATVB, in northern Italy, and Girona, Regicor, in north-eastern Spain) from the Myocardial Infarction Genetics (MIGen) Consortium, ${ }^{12}$ which was accessed through the database of Genotypes and Phenotypes (dbGAP; http://www.ncbi.nlm. nih.gov/gap). Baseline characteristics of the samples used in the study are shown in Table 1.

\section{Polymorphisms and genotyping}

Genomic DNA was extracted from blood cells using a Blood Midi kit (Omega Biotek, Norcross, GA, USA) in accordance with the manufacturer's procedures. DNA samples were genotyped for a set of 384 SNPs using a Custom GoldenGate Panel (Illumina Inc., San Diego, CA, USA). Out of the 384 SNPs, 61 are located in the $1 \mathrm{p} 13.3$ chromosomal region, which spans $150 \mathrm{~kb}$ and includes the CELSR2, PSRC1, $M Y B P H L$, and SORT1 genes; 38 SNPs are in 1q41, which spans $100 \mathrm{~kb}$ and includes the TAF1A, MIA3, and AIDA genes; 159 SNPs are in $9 \mathrm{p} 21$, which spans $300 \mathrm{~kb}$ and comprises the $C D K N 2 A$ and $C D K N 2 B$ genes; and finally, 126 SNPs are in the $10 \mathrm{q} 11$ chromosomal region, which spans $200 \mathrm{~kb}$ and includes the $C X C L 12$ gene.

SNPs were selected as a representative set of the common variation in the four genomic regions according to the following criteria: i) average coverage of 1 SNP every $1.5 \mathrm{~kb}$; ii) minor allele frequency (MAF) higher than 0.05 in European (CEU) HapMap populations; iii) avoiding markers in tight linkage disequilibrium (LD) $\left(r^{2}>0.8\right)$; and iv) giving priority to markers previously reported as associated with CAD. ${ }^{13}$ These criteria were applied to give preference to tag SNPs.

Genotype data coming from the MIGen samples were generated in the corresponding original projects using the Affymetrix 6.0 GeneChip (Affymetrix, Inc., Santa Clara, CA, USA). ${ }^{12}$

\section{Statistical analyses}

Genotyping rate per SNP and individual, Hardy-Weinberg equilibrium (HWE), cryptic relatedness, MAF, and LD were checked using PLINK version 1.07 (Center for Human Genetic Research, Massachusetts General Hospital, and the Broad Institute of Harvard \& Massachusetts Institute of Technology, Cambridge, MA, USA). ${ }^{14}$ Individuals with more than $5 \%$ of missing genotypes were excluded. SNPs with 
genotyping rate lower than 0.95 or with a MAF lower than 5\% were also removed from the analyses.

In order to have the same genetic information, several SNPs not originally genotyped in MIGen samples were imputed simultaneously for cases and controls using $\mathrm{MACH}$ 1.0 software (Department of Biostatistics and Center for Statistical Genetics, University of Michigan, Ann Arbor, MI, USA). ${ }^{15}$ Phased chromosomes from the Tuscan (TSI) samples from the 1000 Genomes Project ${ }^{16}$ were used as a reference panel. A standard single-step imputation approach, with 200 rounds of Markov chain iterations, was used to estimate the crossover maps, error rate maps, and all missing genotypes.

Associations were tested by logistic-regression models adjusted for gender. Permutation tests (1000 permutations) were applied to assess the statistical significance $(P \leq 0.05)$ of the regression models. Permutation procedures provide a computationally intensive approach to empirically generating significance levels. Associations and permutations were calculated using PLINK. ${ }^{14}$ In the case of the European case-control samples, logistic-regression models adjusted for gender were calculated using both the allelic dosage approach, which accounts for imputation uncertainty, and the most likely genotype approach. The mach2dat software version 119 (Department of Biostatistics and Center for Statistical Genetics, University of Michigan) ${ }^{17}$ was used for the dosage approach. Statistical power of the North African samples was checked by the QUANTO software version 1.2 (University of Southern California, Los Angeles, CA, USA). ${ }^{18}$

Haplotype structure and LD pattern analyses were performed by HaploView version 4.2 (Broad Institute) ${ }^{19}$ using the algorithm proposed by Gabriel et al. ${ }^{20}$ Monte Carlo statistical significance was assessed for each chromosomal region in order to evaluate differences between populations in regional LD structure based on the $r^{2}$. This analysis was carried out using 100 random individuals for each different population group (North Africa and southern Europe) using VarLD software version 1.0 (National University of Singapore, Singapore). ${ }^{21}$

Genetic differentiation between Moroccans and Tunisians was calculated by means of a Fisher's exact probability test through Genepop version 4.2 (Laboratiore de Genetique et Environment, Montpellier, France). ${ }^{22}$

Considering that sample sizes of cases and controls from Morocco and Tunisia were not very large, three random effect meta-analyses were performed to provide pooled allelic odd ratios (ORs) with: i) samples from North Africa, ii) samples from southern Europe, and iii) both populations together, paying special attention to markers found as significantly associated in North Africa. Besides the statistical significance, an association was considered positive if the distribution pattern of the risk allele frequency was similar in the different case-control groups. The meta-analyses and the degree of heterogeneity $\left(\mathrm{I}^{2}\right)$ in the different groups analyzed were calculated by means of the Metafor R package (R Foundation for Statistical Computing, Vienna, Austria). ${ }^{23}$ The $\mathrm{I}^{2}$ index describes the percentage of total variation across studies due to true heterogeneity rather than chance. A value of $0 \%$ indicates no observed heterogeneity, and larger values show increasing heterogeneity.

The joint effect of multiple risk SNPs (risk score) was explored by a logistic-regression model that included the number of risk alleles as an independent variable. The increase in risk was quantified by adding supplementary risk alleles according to results of the North African meta-analysis. In this case, homozygotes for the protective allele were coded as 0 , heterozygotes as 1 , and homozygotes for the risk allele were coded as 2. Additionally, the overall score (ie, the number of risk alleles) was calculated for each individual and compared between cases and controls. The ability of this model to discriminate between patients and healthy controls was evaluated through receiver operating characteristic (ROC) curves using logistic-regression models. The area under the ROC curve (AUC) was calculated as a measure of discriminative accuracy. The risk score and the ROC curve were calculated using the PredictABEL R package ${ }^{24}$ (R Foundation for Statistical Computing).

A flowchart of all the analyses performed is included as eFigure 1.

\section{RESULTS}

\section{Genotyping, quality control, and imputation}

According to the above criteria, the initial design included 1 SNP every $1.8 \mathrm{~Kb}$, and the genotyping rate for the 384 SNPs initially tested was $95.7 \%$. However, 15 SNPs were not successfully genotyped, 1 SNP showed a significant departure from Hardy-Weinberg equilibrium after Bonferroni correction, and 19 SNPs failed the frequency test (MAF <0.05); these were excluded from the study. Thus, a total of 349 markers were included in the analyses after quality control: 57 SNPs in 1p13, 36 in 1q41, 148 in 9p21, and 108 in 10q11 (a detailed list can be found in eTable 1). Moreover, one individual was removed for low genotyping rate ( $>5 \%$ of missing genotypes). The statistical power of the Moroccan and Tunisian samples was low when the two samples were analyzed separately. For example, a SNP with a MAF of 0.25 had a statistical power of $37.7 \%$ and $45.8 \%$ for Moroccans and Tunisians, respectively, to detect an OR of 1.6 with a type I error rate of 0.05 . However, when we considered both samples together, the statistical power substantially increased to $70.1 \%$ under the same assumptions as above.

Concerning comparison data from MIGen project, genotyping status and imputation quality index $\left(r^{2}\right)$ are presented in eTable 1. One hundred and seventy-four out of the 349 SNPs were imputed, and only 14 of them showed an imputation quality lower than 0.3 in at least one case-control sample. Therefore, 335 SNPs were considered consistent for this analytical epidemiologic analysis. Most of the imputed 
Table 2. Results of the three meta-analyses performed in North Africans, South Europeans, and merging both continental groups

\begin{tabular}{|c|c|c|c|c|c|c|c|c|c|c|c|c|c|c|}
\hline \multirow{2}{*}{$\mathrm{CHR}$} & \multirow{2}{*}{ BP } & \multirow{2}{*}{ SNP } & \multicolumn{4}{|c|}{ North Africa } & \multicolumn{4}{|c|}{ South Europe } & \multicolumn{4}{|c|}{ All groups } \\
\hline & & & $P$ & OR & $\mathrm{Cl}$ & $1^{2}$ & $P$ & OR & $\mathrm{Cl}$ & $\mathrm{I}^{2}$ & $P$ & OR & $\mathrm{Cl}$ & $1^{2}$ \\
\hline 9 & 22136489 & rs1333051 & 0.016 & 0.470 & $(0.250-0.870)$ & 0 & 0.726 & 1.020 & $(0.900-1.160)$ & 0 & 0.410 & 0.840 & $(0.560-1.260)$ & 74 \\
\hline 9 & 22191189 & rs828576 & 0.038 & 1.530 & $(1.020-2.290)$ & 0 & 0.185 & 0.940 & $(0.860-1.030)$ & 0 & 0.784 & 1.030 & $(0.850-1.230)$ & 39 \\
\hline 10 & 44730995 & rs7907961 & 0.019 & 1.870 & $(1.110-3.140)$ & 0 & 0.851 & 1.010 & $(0.910-1.120)$ & 0 & 0.376 & 1.070 & $(0.920-1.260)$ & 21 \\
\hline 10 & 44786364 & rs800314 & 0.013 & 0.380 & $(0.170-0.810)$ & 0 & 0.410 & 0.870 & $(0.630-1.210)$ & 51 & 0.152 & 0.740 & $(0.490-1.120)$ & 56 \\
\hline 10 & 44855663 & rs 266105 & 0.007 & 2.180 & $(1.240-3.850)$ & 0 & 0.729 & 0.980 & $(0.860-1.110)$ & 0 & 0.245 & 1.260 & $(0.850-1.860)$ & 70 \\
\hline 10 & 44856370 & rs266103 & 0.019 & 1.890 & $(1.110-3.220)$ & 0 & 0.601 & 0.970 & $(0.850-1.100)$ & 0 & 0.369 & 1.150 & $(0.850-1.54)$ & 53 \\
\hline 10 & 44861220 & rs7918568 & 0.047 & 1.600 & $(1.010-2.530)$ & 0 & 0.300 & 1.060 & $(0.950-1.180)$ & 0 & 0.144 & 1.080 & $(0.970-1.200)$ & 0 \\
\hline
\end{tabular}

$\mathrm{Cl}$, confidence interval; $\mathrm{I}^{2}$, index to quantify the $\%$ of true heterogeneity (total heterogeneity/total variability); OR, odds ratio.

SNPs $(79 \%)$ showed high accuracy $\left(r^{2}>0.75\right)$, indicating good quality of the imputation performed. After quality control per individual, a total of 4979 case-control samples from Europe were used for comparisons, with 3352 individuals from the ATVB (Italy) and 627 from the Regicor (Spain) studies.

\section{Marker association analyses}

A total of 5 SNPs were associated with CAD in Morocco (2 in the $1 \mathrm{p} 13$ chromosomal region and 1 in each one of the other three regions), whereas 26 were associated with CAD in Tunisia ( 5 in the 1 p13 region, 7 in the 9 p 21 , and 14 in the $10 \mathrm{q} 11$ region). Logistic regression results and genetic effects of these SNPs are shown in eTable 2. None of these SNPs was associated independently in Morocco and Tunisia, but most of them (24 out of 31) showed the same trends in the two African populations (ie, OR values higher or lower than 1).

Preliminary comparisons of the results in North Africa with the two European samples from the MIGen Consortium pointed out a low replicability of association signals between both groups of populations (eTable 2). For example, 12 out of 26 of the SNPs associated in the Tunisian sample were also associated in the ATVB study, but 4 of them showed an opposite effect.

An exact test indicated consistent genetic similarity between the two North African countries for both cases $(P=0.248)$ and controls $(P=0.927)$. In the meta-analysis of North African samples, seven SNPs showed significant associations with a remarkably low heterogeneity $\left(\mathrm{I}^{2}=0 \%\right.$ ) (Table 2$)$, with two corresponding to the $9 \mathrm{p} 21$ region and five corresponding to the $10 \mathrm{q} 11$ region. All of these SNPs were located in intergenic regions. In the $1 \mathrm{p} 13$ and $1 \mathrm{q} 41$ regions, no association was detected.

The potential population specificity of the seven risk variants detected in North Africa was assessed by comparison with the results of the meta-analyses carried out in the European populations. None of these seven SNPs showed significant associations in southern European populations (Table 2). The heterogeneity among the southern European samples was remarkably low, except for the rs800314 marker $\left(\mathrm{I}^{2}=51 \%\right)$.
The third meta-analysis performed using the North Africans and the southern Europeans case-control samples did not show significant associations for the seven SNPs previously identified in the African meta-analyses. The heterogeneity among African and European samples was medium to high ( $\mathrm{I}^{2}$ from $21 \%$ to $74 \%$ ), except for the rs7918568 marker $\left(\mathrm{I}^{2}=0 \%\right)$ (Table 2).

\section{Haplotype analyses}

Haplotype structure was analyzed to better understand the patterns of associations observed in these two different populations groups. As expected, different patterns of haplotype blocks were identified in North Africa and southern Europe (LD patterns and haplotype block structure are plotted in eFigure 2A, eFigure $2 \mathrm{~B}$, eFigure $2 \mathrm{C}$, and eFigure 2D). In general, a higher number of haplotype blocks in North Africans compared to southern Europeans were observed in all four chromosomal regions: 6 versus 2 blocks in 1p13; 4 versus 2 in 1q41; 23 versus 21 in 9p21; and 12 versus 10 in $10 \mathrm{q} 11$. The two population groups showed statistically significant differences in LD patterns for all four chromosomal regions $(P<0.001$ for the $1 \mathrm{p} 13$ and $9 \mathrm{p} 21$ regions, and $P<0.05$ for the $1 \mathrm{q} 41$ and $10 \mathrm{q} 11$ genomic regions). The comparison of the haplotype blocks that included the seven African risk SNPs indicated that these risk variants are not located in the same haplotype blocks in African and European individuals (eFigure 2C and eFigure 2D).

\section{Risk scores}

The genomic risk-score was calculated using the information of SNPs associated in the North African meta-analysis. After applying LD pruning criteria in both European and North African samples $\left(r^{2}<0.4\right.$ ), six SNPs (making possible risk scores from 0 to 12 risk alleles) were considered in the analysis (eTable 3). In North Africa, the distribution of the risk score between patients and healthy individuals showed significant differences (Figure 1). A total of $32 \%$ of cases carried 6 risk alleles, whereas only $26 \%$ of controls showed the same amount of risk alleles. The risk score applied in North Africa showed a remarkable ability to discriminate between individuals with and without the disease (AUC $=0.63 ; 95 \% \mathrm{CI}, 0.56-0.69$ ) 


\section{Distribution of Risk Score}

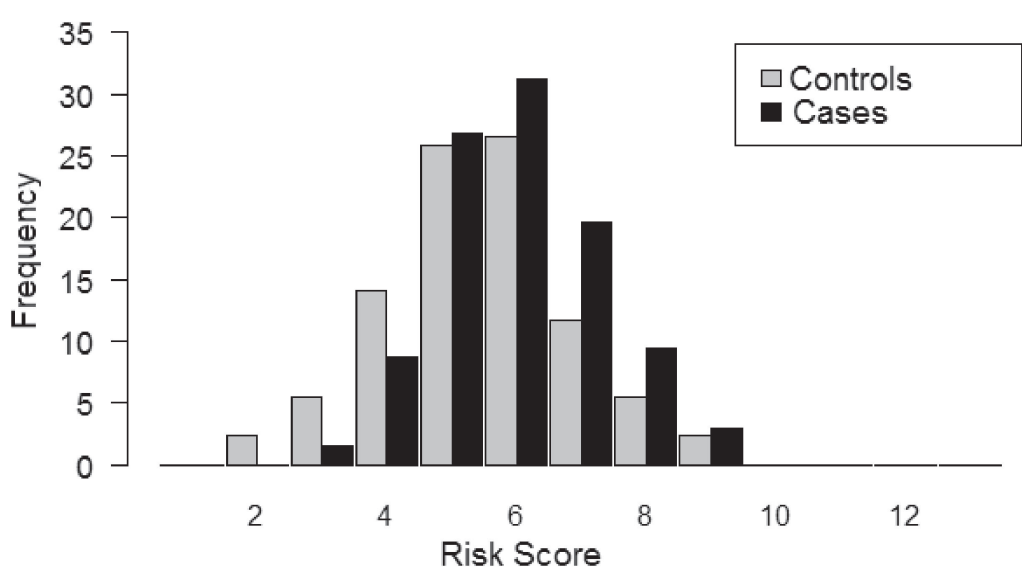

Figure 1. Distribution of North African individuals according to the number of risk alleles in the sample of cases and controls analyzed.

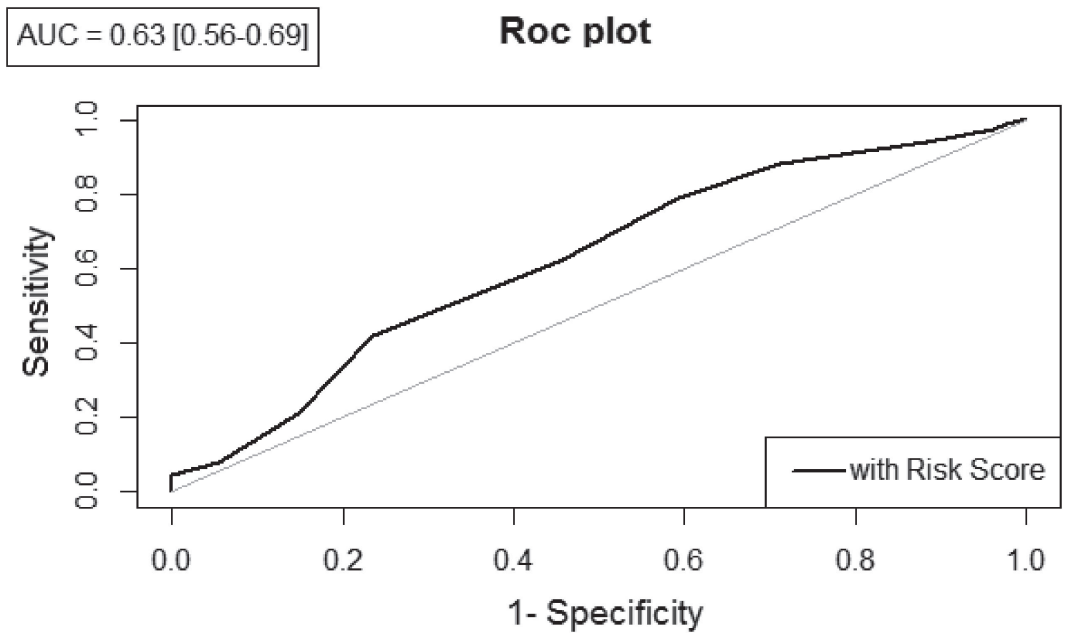

Figure 2. Receiver operating characteristic (ROC) curves in North Africa. AUC, Area Under the ROC Curve with 95\% confidence interval.

(Figure 2). In addition, the risk for CAD in North Africa increases with the number of risk alleles included in the risk score. The susceptibility to develop CAD in North Africans shows a significant value from the group that had 6 risk alleles $(P=0.04)$ and, as the number of risk alleles increases, the OR value also increases, reaching a maximum for the individuals with 8-12 risk alleles (Figure 3). Comparatively, this risk score had remarkably lower ability to predict CAD risk in southern Europeans than in North Africans $(\mathrm{AUC}=0.52 ; 95 \%$ CI, 0.50-0.54) (eFigure 3).

\section{DISCUSSION}

This study provides novel genetic data of epidemiological interest in North Africa. The genomic variation of four CAD risk regions $(1 \mathrm{p} 13,1 \mathrm{q} 41,9 \mathrm{p} 21$, and $10 \mathrm{q} 11)$ was analyzed through the genotyping of 384 SNPs in two North African case-control samples, specifically from Morocco and Tunisia.

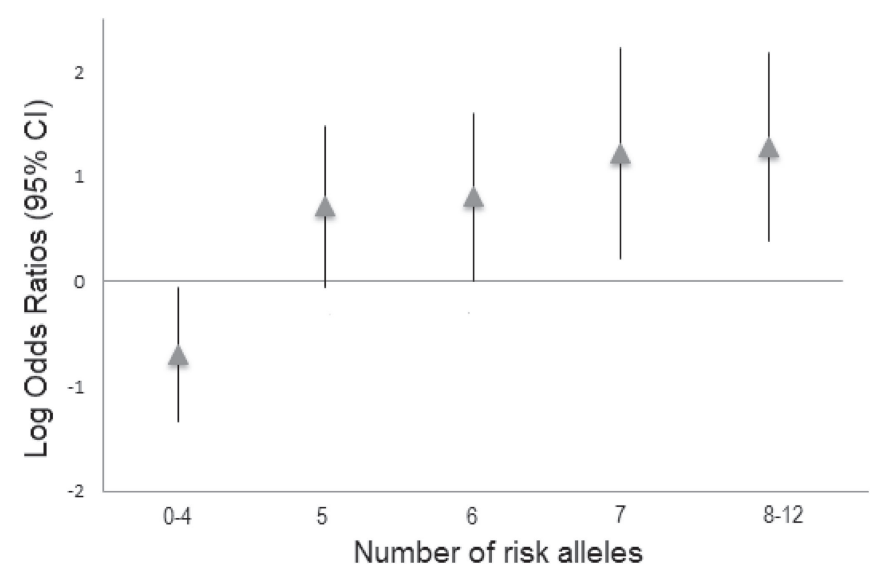

Figure 3. Logarithm of the odds ratios with $95 \%$ confidence interval for CAD (adjusted by gender) in North African individuals carrying increasing numbers of risk alleles. 
Previous studies of these genomic regions were based on populations of European descent. To the best of our knowledge, these are the first results in populations from Africa. Consequently, this study aimed to assess whether findings about CAD risk variants in European populations can also be extended to North Africans.

We found significant association signals in the four genomic regions analyzed also in Moroccan and Tunisian samples. However, in the meta-analyses performed with the African case-control samples, only the 9p21 and 10q11 regions showed association signals. None of the North African risk SNPs showed association in European samples. In addition, the calculated risk model had a remarkable ability to discriminate cases and controls in Africa $(\mathrm{AUC}=0.63)$ but not in Europe $(\mathrm{AUC}=0.52)$. These arguments suggest the existence of continental-specific CAD risk variants in these chromosomal regions.

\section{Continent-specific risk markers within global risk regions}

Among the several CAD risk loci identified so far, the 9p21 locus has a prominent position because of the impressive robustness of the association results. ${ }^{25-27}$ In the North African meta-analysis, two SNPs in this genomic region showed association with CAD. A detailed analysis of the literature revealed that the rs 1333051 marker was previously associated with type 2 diabetes in Mexican Americans. ${ }^{28}$ The $10 \mathrm{q} 11$ genomic region shows the highest number of associated SNPs in North Africa (5 out of 7 of the associated SNPs). None of these five SNPs was found to be associated with CAD in previous studies.

Regarding the logistic association performed in the four populations analyzed (Morocco, Tunisia, Italy, and Spain), the associated SNPs were not the same across populations, even though association signals were present in all of the regions studied. Of note, some associated SNPs are close to SNPs associated in other populations, while other SNPs are population-specific (ie, physically distant or not in LD with SNPs associated in other populations).

The number of SNPs associated with risk of CAD in southern Europe is considerably larger than in North Africa, as expected from the much larger sample size (and statistical power) of the European dataset. The ability of the limited sample size to reach the significance threshold of the North African samples may be the cause of the low number of associations found in Africa and the lack of replication in African samples of most associations found in Europe. Still, the risk SNPs found in North Africa but not replicated in Europe underline the possible role of the genomic architecture in these results. Statistical power calculations indicate reduced power of the North African case-control samples to detect small genetic effects, but adequate power to detect a risk with an $\mathrm{OR} \geq 1.6$. Although sample size biases cannot be fully discounted, the SNPs associated in Africa but not in Europe show an adequate statistical consistency according to the permutation tests performed.

The significant differences $(P<0.05)$ in the LD and the haplotype patterns in the two population groups (eFigure $2 \mathrm{~A}$, eFigure 2B, eFigure 2C, and eFigure 2D) confirm the heterogeneity in the genomic structure between North Africans and Europeans. This general trend of lower haplotype diversities and higher LD values when the geographic distance from Africa increases has been previously observed. ${ }^{29,30}$ Further, the haplotype blocks containing risk SNPs differ between North Africa and southern Europe. This situation is especially evident in the 10q11 region, where three out of the five associated SNPs are not located in any block in North Africa, while in Europe only one of these SNPs is not located in a block (eFigure 2D). Differences in allele frequency and LD patterns may be related to the observed differences in the association tests. Higher LD (illustrated by the different number of haplotype blocks) in populations of European origin compared to Africans reduces the likelihood of finding a substantial number of associated SNPs in North Africa. ${ }^{31}$ African populations are genetically more diverse than European and Asian populations, a finding that is in agreement with the Recent-African-Origin hypothesis. ${ }^{32}$ Consequently, replication studies across European populations have been largely successful because of the general genetic similarity across European populations. A failure to replicate an association signal in Africa may be influenced by variations in LD patterns between European and African populations. ${ }^{31}$ Low levels of LD reduce the correlation among casual variants and nearby SNPs. As a consequence, the probability of detecting association signals is lower among Africans than in Europeans if the causal variants are not assayed directly.

The potential existence of ethnicity-specific CAD risk markers is not an isolated case; rather, such risk markers are common in complex diseases. A recent study detected significant differences in association signals among African and European populations screening the catalog of all published GWAS curated at the National Human Genome Research Institute website. ${ }^{33}$ This work stressed a modest correlation in the risk allele frequencies between Europeans and Africans. The point estimates of risk were opposite in direction or differed more than 2-fold in 79\% of the comparisons between European and African groups. Along with this fact, the under-representation of non-European population in GWAS suggests the need to extend epidemiological studies to a much broader ensemble of populations, including ethnic minorities. ${ }^{3}$

\section{Conclusion}

This study shows that in North African populations, as in European populations, the regions $1 \mathrm{p} 13,1 \mathrm{q} 41,9 \mathrm{p} 21$, and $10 \mathrm{q} 11$ contain genetic risk variants associated with CAD. However, Europeans and North Africans do not always share 
the same risk variants. The differences obtained in CAD risk variants between Europeans and North Africans suggest that findings from one population cannot be directly applied to populations from other continents. That is, differences in the genomic architecture due to different demographic histories should be taken into account in epidemiological studies of complex disorders. This preliminary evidence from four CAD risk genomic regions in North African populations needs to be extended in additional larger analyses.

\section{ONLINE ONLY MATERIALS}

eTable 1. Genomic location of the genetic variants, genotyping, and imputation details.

eTable 2. Case-control allelic frequencies and association parameters adjusted for gender in the genotype and imputed case-control samples.

eTable 3. Genomic location of the 6 North African SNPs used to calculate the risk score in the case-control samples.

eFigure 1. Flowchart of all the analyses performed.

eFigure 2A. Linkage disequilibrium patterns and haplotype block structure observed in North Africa and in southern Europe for the region $1 \mathrm{p} 13$.

eFigure 2B. Linkage disequilibrium patterns and haplotype block structure observed in North Africa and in southern Europe for the region 1q41.

eFigure 2C. Linkage disequilibrium patterns and haplotype block structure observed in North Africa and in southern Europe for the region 9p21.

eFigure 2D. Linkage disequilibrium patterns and haplotype block structure observed in North Africa and in southern Europe for the region $10 \mathrm{q} 11$.

eFigure 3. Receiver operating characteristic (ROC) curves in southern Europe.

\section{ACKNOWLEDGMENTS}

The authors declare that the work has been performed according to the ethical principles set out in the Declaration of Helsinki. This research was supported by the Ministerio de Ciencia e Inovación CGL2011-27866 project to PM, and the Predoctoral Master and Back Grant (AF-DR-A2011B-48666$25399 / 2011)$ to DZ. We want to thank all volunteers for sample donation.

Conflicts of interest: None declared.

\section{REFERENCES}

1. Wilson PW, D'Agostino RB, Levy D, Belanger AM, Silbershatz H, Kannel WB. Prediction of coronary heart disease using risk factor categories. Circulation. 1998;97:1837-47.

2. Girelli D, Martinelli N, Peyvandi F, Olivieri O. Genetic architecture of coronary artery disease in the genome-wide era: implications for the emerging "golden dozen" loci. Semin
Thromb Hemost. 2009;35(7):671-82.

3. Bustamante CD, Burchard EG, De la Vega FM. Genomics for the world. Nature. 2011;475:163-5.

4. Adler J, Rangwalla SC, Dwamena BA, Higgins PD. The prognostic power of the NOD2 genotype for complicated Crohn's disease: a meta-analysis. Am J Gastroenterol. 2011;106: 699-712.

5. Lesage S, Zouali H, Cézard JP, Colombel JF, Belaiche J, Almer $\mathrm{S}$, et al. CARD15/NOD2 mutational analysis and genotypephenotype correlation in 612 patients with inflammatory bowel disease. Am J Hum Genet. 2002;70:845-57.

6. Hama I, Ratbi I, Reggoug S, Elkerch F, Kharrasse G, Errabih I, et al. Non-association of Crohn's disease with NOD2 gene variants in Moroccan patients. Gene. 2012;499:121-3.

7. Zouiten-Mekki L, Zaouali H, Boubaker J, Karoui S, Fekih M, Matri S, et al. CARD15/NOD2 in a Tunisian Population with Crohn's Disease. Dig Dis Sci. 2005;50:130-5.

8. Liu Y, Hauser MA, Akafo SK, Qin X, Miura S, Gibson JR, et al. Investigation of known genetic risk factors for primary open angle glaucoma in two populations of African ancestry. Invest Ophthalmol Vis Sci. 2013;54:6248-54.

9. Cauchi S, Ezzidi I, El Achhab Y, Mtiraoui N, Chaieb L, Salah D, et al. European genetic variants associated with type 2 diabetes in North African Arabs. Diabetes Metab. 2012;38:316-23.

10. Wojczynski MK, Li M, Bielak LF, Kerr KF, Reiner AP, Wong $\mathrm{ND}$, et al. Genetics of coronary artery calcification among African Americans, a meta-analysis. BMC Med Genet. 2013; $14: 75$.

11. Ni X, Zhang J. Association between 9p21 genomic markers and ischemic stroke risk: evidence based on 21 studies. PLoS One. 2014;9:e90255.

12. Myocardial Infarction Genetics Consortium, Kathiresan S, Voight BF, Purcell S, Musunuru K, Ardissino D, Mannucci $\mathrm{PM}$, et al. Genome-wide association of early-onset myocardial infarction with single nucleotide polymorphisms and copy number variants. Nat Genet. 2009;41:334-41.

13. Roberts R. Genetics of coronary artery disease: an update. Methodist Debakey Cardiovasc J. 2014;10:7-12.

14. Purcell S, Neale B, Todd-Brown K, Thomas L, Ferreira MA, Bender D, et al. PLINK: a tool set for whole-genome association and population-based linkage analyses. Am J Hum Genet. 2007;81:559-75.

15. Li Y, Willer CJ, Ding J, Scheet P, Abecasis GR. MaCH: using sequence and genotype data to estimate haplotypes and unobserved genotypes. Genet Epidemiol. 2010;34:816-34.

16. 1000 Genomes Project Consortium, Abecasis GR, Altshuler D, Auton A, Brooks LD, Durbin RM, Gibbs RA, et al. A map of human genome variation from population-scale sequencing. Nature. 2010;467:1061-73.

17. Li Y, Willer C, Sanna S, Abecasis G. Genotype imputation. Annu Rev Genomics Hum Genet. 2009;10:387-406.

18. Gauderman WJ. Sample size requirements for association studies of gene-gene interaction. Am J Epidemiol. 2002;155:478-84.

19. Barrett JC, Fry B, Maller J, Daly MJ. Haploview: analysis and visualization of LD and haplotype maps. Bioinformatics. 2005;21:263-5.

20. Gabriel SB, Schaffner SF, Nguyen H, Moore JM, Roy J, Blumenstiel B, et al. The structure of haplotype blocks in the 
human genome. Science. 2002;296:2225-9.

21. Ong RT, Teo YY. varLD: a program for quantifying variation in linkage disequilibrium patterns between populations. Bioinformatics. 2010;26:1269-70.

22. Rousset F. Genepop'007: a complete reimplementation of the Genepop software for Windows and Linux. Mol Ecol Resour. 2008;8:103-6.

23. Viechtbauer W. Conducting meta-analyses in $\mathrm{R}$ with the metafor package. J Stat Softw. 2010;36:1-48.

24. Kundu S, Aulchenko YS, van Duijn CM, Janssens AC. PredictABEL: an $\mathrm{R}$ package for the assessment of risk prediction models. Eur J Epidemiol. 2011;26:261-4.

25. Assimes TL, Knowles JW, Basu A, Iribarren C, Southwick A, Tang H, et al. Susceptibility locus for clinical and subclinical coronary artery disease at chromosome $9 \mathrm{p} 21$ in the multi-ethnic ADVANCE study. Hum Mol Genet. 2008;17:2320-8.

26. Meng W, Hughes AE, Patterson CC, Belton C, Kee F, McKeown PP. Chromosome 9p21.3 is associated with earlyonset coronary heart disease in the Irish population. Dis Markers. 2008;25:81-5.

27. Schunkert H, Götz A, Braund P, McGinnis R, Tregouet DA, Mangino M, et al. Europe PMC Funders Group. Repeated replication and a prospective meta-analysis of the association between chromosome 9p21.3 and coronary artery disease. Circulation. 2008 Apr 1;117(13):1675-84.

28. Parra EJ, Below JE, Krithika S, Valladares A, Barta JL, Cox NJ, et al. Genome-wide association study of type 2 diabetes in a sample from Mexico City and a meta-analysis of a MexicanAmerican sample from Starr County, Texas. Diabetologia. 2011;54:2038-46.

29. Henn BM, Botigué LR, Gravel S, Wang W, Brisbin A, Byrnes JK, et al. Genomic ancestry of North Africans supports back-toAfrica migrations. PLoS Genet. 2012;8:e1002397.

30. Botigué LR, Henn BM, Gravel S, Maples BK, Gignoux CR, Corona E, et al. Gene flow from North Africa contributes to differential human genetic diversity in southern Europe. Proc Natl Acad Sci USA. 2013;110:11791-6.

31. Teo YY, Small KS, Kwiatkowski DP. Methodological challenges of genome-wide association analysis in Africa. Nat Rev Genet. 2010;11:149-60.

32. Disotell TR. Archaic human genomics. Am J Phys Anthropol. 2012;149 Suppl 55:24-39.

33. Ntzani EE, Liberopoulos G, Manolio TA, Ioannidis JP. Consistency of genome-wide associations across major ancestral groups. Hum Genet. 2012;131:1057-71. 